\title{
Nuevas tecnologías para la ingeniería civil en un entorno de realidad virtual: aplicación didáctica integrada de SIG y BIM
}

\author{
Víctor Martínez-Ibáñez ${ }^{a}$, Isidro Cantarino ${ }^{b}$, José Ángel Aranda Domingo ${ }^{c}$, Fco. Javier Camacho \\ Torregrosa $^{\mathrm{d}}$ \\ a'Departamento de Ingeniería del Terreno, Universitat Politècnica de València. Email: vicmarib@trr.upv.es; \\ ${ }^{b}$ Departamento de Ingeniería del Terreno, Universitat Politècnica de València. Email: icantari@trr.upv.es \\ ${ }^{c}$ Departamento de Ingeniería Gráfica, Universitat Politècnica de València. Email: jaranda@dig.upv.es; \\ ${ }^{\mathrm{d}}$ Departamento de Ingeniería e Infraestructura de los Transportes, Universitat Politècnica de València. Email: \\ fracator@tra.upv.es
}

\begin{abstract}
In recent years, both teachers and professionals are facing a growing role of assisted design software at all levels of applied science. Specifically, the design of construction projects (such as road geometric design) is essential in the field of civil engineering, since it requires optimization of resources, cost savings, early detection of errors and reduction of environmental impacts. Two fundamental tools are postulated for this purpose, such as SIG (Geographic Information System) and BIM (Building Information Modeling), whose mastery by students multiplies their employability in the civil engineering industry. In this work we want to show the synergy obtained by the application of these two methodologies in the Bachelor's Degree in Geomatic and Surveying Engineering, its implementation in the subject "Civil Engineering, and the advantages of using a virtual reality environment as a teaching tool for solving educational exercises on a real basis.
\end{abstract}

Keywords: GIS, BIM, linear works design, layout optimization, teaching application, virtual reality

\section{Resumen}

En los últimos años, tanto docentes como profesionales estamos asistiendo a un creciente protagonismo del software de diseño asistido en todos los niveles de la ciencia aplicada. En concreto, el diseño de proyectos constructivos (como el diseño geométrico de carreteras) es esencial en el ámbito de la ingeniería civil, puesto que requiere optimizar recursos, economizar costes, detección temprana de errores y reducir afecciones al medio ambiente. Se postulan dos herramientas fundamentales para este fin, como son los softwares de SIG (Sistema de Información Geográfica) y BIM (Building Information Modeling), cuyo dominio por parte del alumnado multiplica su empleabilidad en la industria de la ingeniería civil. En este trabajo se quiere mostrar la sinergia obtenida por la aplicación de estas dos metodologías en el Grado de Ingeniería en Geomática y Topografia, su puesta en marcha en la asignatura "Ingeniería civil", y las ventajas del empleo de un entorno de realidad virtual como herramienta docente para la resolución de ejercicios didácticos sobre base real.

Palabras clave: SIG, BIM, diseño obras lineales, optimización trazado, aplicación docente, realidad virtual 


\section{Introducción}

\subsection{Sistemas de Información Geográfica (SIG)}

Los Sistemas de Información Geográfica (SIG) son herramientas bien conocidas y empleadas por los gestores del territorio, pues permiten organizar, manipular y analizar grandes cantidades de datos de muy distinta índole con la única exigencia de estar geográficamente referenciados. Los softwares que desarrollan esta herramienta comenzaron a implantarse a finales del siglo pasado y sus procedimientos han formado parte obligatoria de los planes de estudio de los alumnos de cartografía prácticamente desde entonces.

En concreto, en el ámbito de la ingeniería civil, un SIG permite ordenar, clasificar y evaluar las características del terreno sobre el que se va a desarrollar una determinada infraestructura. Si el objetivo es reducir costes económicos y ambientales, el empleo de un SIG facilita mucho el trabajo previo de estudio y valoración de un proyecto. Por lo tanto, justifica sobradamente la inserción de este software en las prácticas de la asignatura.

\subsection{Building Information Modeling (BIM)}

Building Information Modeling (BIM) es una metodología de trabajo colaborativa para la gestión de proyectos de edificación y obra civil a través de una preconstrucción digital de las obras. Este modelo conforma una gran base de datos que permite gestionar los elementos que forman parte de la infraestructura durante todo el ciclo de vida de esta (Gosalves-López et al. 2016). El modelo BIM es un repositorio único con información ordenada, coordinada, coherente y compartida, que se actualiza en tiempo real durante las fases de la obra: proyecto, construcción y explotación. Esto implica un gran ahorro de tiempo (no es necesario convertir o interpretar desde varios tipos de archivo), así como un menor número de errores (además de su identificación más sencilla, la menor necesidad de intercambios de información reduce la frecuencia de errores).

La implantación de la metodología BIM aplicada a la ingeniería de carreteras es todavía incipiente (Bradley et al. 2016). Sin embargo, la demanda para la implantación de BIM por parte del sector es creciente (Gómez-Muñoz et al. 2017; es.BIM 2019), y muy pronto será obligatorio en licitaciones públicas (Ministerio de la Presidencia 2019), por lo que la demanda de personal formado en el uso de herramientas específicas es creciente año a año.

Por todo lo anterior, han surgido iniciativas por parte de algunas instituciones educativas para su instauración en los planes de estudio (Hu 2019). La Escuela de Caminos de la UPV ha sido pionera en este aspecto, donde BIM se integra como asignaturas optativas de Grado y Máster, destacando por ser de las más demandadas (Camacho-Torregrosa et al. 2019).

\subsection{Integración de SIG y BIM}

La combinación entre SIG y BIM es el punto de partida de la innovación que se propone en el presente trabajo. Se parte de la siguiente premisa: el flujo de trabajo BIM puede optimizarse mediante un análisis preliminar utilizando herramientas SIG. Así, mediante SIG puede estudiarse diferentes alternativas para el corredor de la futura traza de la carretera. Características tales como la geología, usos y ocupación del terreno, pendientes, aspectos ambientales, etc., pueden ser tratados con anterioridad desde un SIG con objeto de facilitar un diseño de trazado óptimo que minimice costes económicos y ambientales.

Sin embargo, SIG no es una herramienta de diseño de carreteras. Por ello, se plantea a continuación el empleo del BIM para ajustar y cuantificar las características constructivas del proyecto como, p.ej., el 
volumen del movimiento de tierras, o garantizar el cumplimiento de los parámetros geométricos normativos y de seguridad vial (Ministerio de Fomento 2016). En un posterior estudio de alternativas, estos datos numéricos acaban por objetivar la decisión final de selección de la alternativa más apropiada.

Debe destacarse el carácter innovador del flujo de trabajo presentado: una de las principales limitaciones para el desarrollo óptimo de BIM en infraestructuras es la falta de interoperabilidad entre software BIM de diferente naturaleza (Martinez-Ibañez et al. 2017). Mediante esta experiencia, se da un paso adelante al enseñar técnicas a los alumnos para vencer, al menos en parte, la mencionada limitación. Por otra parte, los docentes optaron por el uso de un software de modelado BIM que emplea un entorno de realidad virtual, buscando incrementar la motivación y la comprensión de los supuestos prácticos por parte del alumnado. En relación con lo anterior, debe tenerse en cuenta que la realidad virtual aplicada a la metodología BIM es un campo con un enorme potencial (Charef et al. 2018; Sidani et al. 2019), que puede multiplicar la empleabilidad de los alumnos.

En la Fig. 1 se ilustra sobre las conexiones de la información geográfica aportada y su gestión desde los diferentes tipos de software.

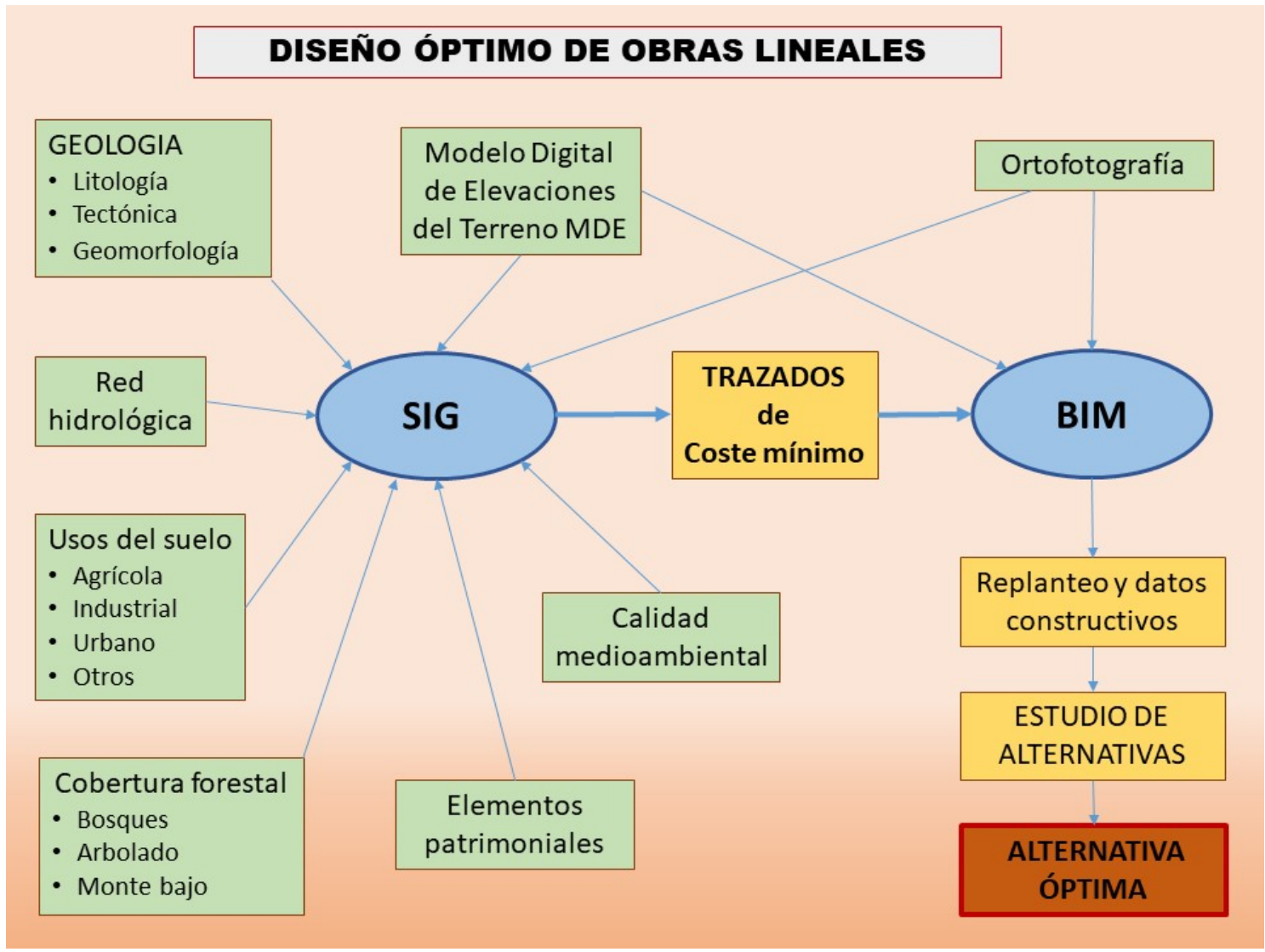

Fig. 1. Diagrama de flujo para el diseño óptimo del trazado de obras lineales

\subsection{Situación docente en ETSIGCT}

Los conceptos y planteamientos de la Ingeniería Civil en sus diferentes tipos de actuaciones (obras lineales, obras hidráulicas, obras marítimas, etc.) se desarrollan actualmente en la ETSIGCT (Escuela Técnica Superior de Ingeniería Geodésica, Cartográfica y Topográfica) de la UPV mediante la asignatura troncal homónima emplazada en el 3er. Curso del Grado de Ingeniería en Geomática y Topografía (1er. Cuatrimestre y 4,5 créditos). Una proporción importante de los egresados de esta Escuela tienen como 
destino laboral empresas de consultoría de ingeniería, y constructoras dentro del ámbito de la obra civil, lo que pone de relieve la importancia de esta asignatura en el plan de estudios.

Siendo que su temario y conceptos teóricos están bien delimitados, es en las prácticas de esta asignatura donde se debe aportar un acercamiento a la realidad profesional, mediante técnicas y herramientas actualizadas que consigan vitalizar su desarrollo. Con este objetivo, se ha planteado la inclusión coordinada de GIS y BIM, dos potentes herramientas de software que se presentan imprescindibles en el desarrollo profesional de proyectos de infraestructuras de ingeniería civil.

Como ya se ha indicado, la asignatura "Ingeniería Civil" se sitúa en el 3er. Curso del Grado de Ingeniería en Geomática y Topografía. En ese momento, los alumnos ya han recibido ( $2^{\circ}$ curso) una formación generalista sobre utilización de software comercial de Sistemas de Información Geográfica (como es el ArcGis ${ }^{\circledR}$ de ESRI) en una asignatura homónima de 6 créditos. Por otro lado, reciben paralelamente también en el 1er cuatrimestre del 3er. curso una formación práctica y aplicada de replanteo de obras lineales en la asignatura de Topografía de Obras (7,5 créditos).

En ambos precedentes no se trata la planificación generalista de obras lineales, la definición de trazado minimizando el coste y su visualización espacial o 3D, aspectos primordiales en los estudios de soluciones y anteproyectos necesarios en el proceso constructivo real.

Los actuales planes de estudio de la titulación de grado en la ETSIGCT no incluyen la formación en esta metodología BIM. Como se ha mencionado anteriormente, es preciso destacar que la legislación vigente obligará al uso de este tipo de metodología en el proyecto, construcción y mantenimiento de infraestructuras, pues permite acercar las tecnologías de la información y de la comunicación al sector de la construcción, lo que se traduce en múltiples ventajas. Esta cuestión justifica plenamente la inclusión de un primer encuentro con la metodología BIM en la Escuela.

Por otro lado, mientras que el comportamiento de la asignatura "Ingeniería Civil" desde la óptica de las encuestas de opinión del alumnado recibe unas valoraciones favorables en su apartado teórico, no lo es tanto en el práctico. En concreto, en su apartado "La metodología empleada y las actividades realizadas en la asignatura ayudan a aprender al alumnado" tan solo se recibe una calificación de 5,5 en el último curso encuestado. Es un punto débil que claramente hay que mejorar y obliga a replantear necesariamente el desarrollo de las prácticas.

\section{Objetivos}

En esta comunicación se detallará el uso integrado de herramientas BIM y SIG a través de un entorno de realidad virtual, como innovación docente incorporada a las prácticas realizadas en la asignatura "Ingeniería Civil", del 3er. Curso del Grado de Ingeniería en Geomática y Topografía a lo largo del curso 2019-2020.

Los objetivos de la innovación docente pasan por mitigar la total falta de contenidos BIM impartidos en la ETSIGCT, y mejorar las habilidades de los alumnos en el manejo de herramientas SIG, donde se vienen observando algunas carencias. La anterior situación no es deseable en un contexto de demanda creciente de profesionales formados en este tipo de técnicas. Además, la innovación planteada avanza en la interoperabilidad entre software de distintas naturalezas, aspecto éste en plena fase de investigación y desarrollo en la industria de la construcción, lo que permite a los alumnos estar en la punta de lanza de estas tecnologías. Por último, el empleo de un entorno de realidad virtual para el diseño de carreteras busca provocar un mayor interés por las prácticas y mejorar su valoración por parte del alumnado. 


\section{Desarrollo de la innovación}

\subsection{Planteamiento general}

La innovación planteada se articula en dos partes bien definidas relacionadas con el software empleado y los objetivos de cada una de ellas. Esta innovación ya se ha comenzado a impartir en el curso 2019-2020, por lo que se dispone de unos primeros resultados.

En una primera parte fundamental, los alumnos deben recordar y aplicar sus conocimientos SIG y tratar datos descriptivos del terreno de acceso público. El objetivo es obtener una ruta óptima de acceso viario a partir de dos alternativas planteadas. La caracterización del terreno se consigue mediante el aporte de los datos morfológicos georreferenciados disponibles en webs oficiales. En concreto, se trata de los repositorios del Instituto Geográfico Nacional (IGN), Instituto Cartográfico Valenciano (ICV) e Instituto Geológico y Minero de España (IGME). Estos son:

\section{Modelo de Elevación del Terreno (MDT) $5 \times 5$ m, del ICV o IGN (formato ráster)}

Sistema de Información de Ocupación del Suelo en España (SIOSE), de 25m de resolución, del IGN (formato vectorial)

Geocronología y estratigrafía (tipos litológicos) provenientes del Mapa Geológico Nacional (MAGNA) escala 1:50.000 del IGME (formato vectorial).

- Espacios Naturales protegidos: Parques Naturales y Red Natura 2000, del ICV (formato vectorial); cauces y riberas según el Plan de Acción Territorial sobre Riesgos de Inundación en la Comunidad Valenciana (PATRICOVA) disponible en ICV.

- Ortofotos del Plan Nacional de Ortofotografía Aérea (PNOA) del IGN, mediante conexión a su servidor remoto Web Map Service (WMS).

La ruta óptima debe satisfacer un coste mínimo estimado atendiendo a los anteriores factores: pendiente del terreno, facilidad de excavación de los materiales geológicos, valor del tipo de suelo ocupado (y expropiaciones) y afección mínima a espacios naturales protegidos. Como se indicará más adelante, al alumno se le plantea un problema de conexión viaria entre dos puntos que es único para cada alumno y por tanto debe desarrollar individualmente.

Como finalidad paralela, se plantea el trabajo mediante el manejo de un software SIG de instalación libre como es el QGIS. Se trata de una perspectiva complementaria al software comercial de ESRI (ArcGis ${ }^{\circledR}$ ) que ya conocen y que les puede abrir numerosas expectativas en oficinas técnicas que no se puedan permitir el pago de licencias de alto coste.

El segundo bloque del proyecto consiste en trasladar la traza obtenida mediante SIG a un software BIM como es el Infraworks de Autodesk ${ }^{\circledR}$. Permite una visualización espacial de la carretera, así como detallar sus principales características de alzados, transversales, movimientos de tierras (desmontes/terraplenes), etc. Todo ello se realiza a través de un entorno realista (realidad virtual), que motiva y facilita la comprensión del alumno (Fig. 2).

El uso de herramientas BIM permite al alumno refinar el trabajo realizado en SIG, incorporando así criterios de trazado geométrico de carreteras, y de optimización de movimientos de tierras. Todo ello conduce al alumno a dar un importante paso adelante en su formación profesional, pues acerca su propuesta hasta una realidad constructiva más cercana a los diseños que realmente se desarrollan en el sector.

Se persigue, por tanto, complementar la visión geomática que domina en el alumnado, con un enfoque complementario de la ingeniería civil. Además, incorpora el criterio ingenieril, acercándose al carácter multidisciplinar que presenta actualmente el sector en su actividad cotidiana. 


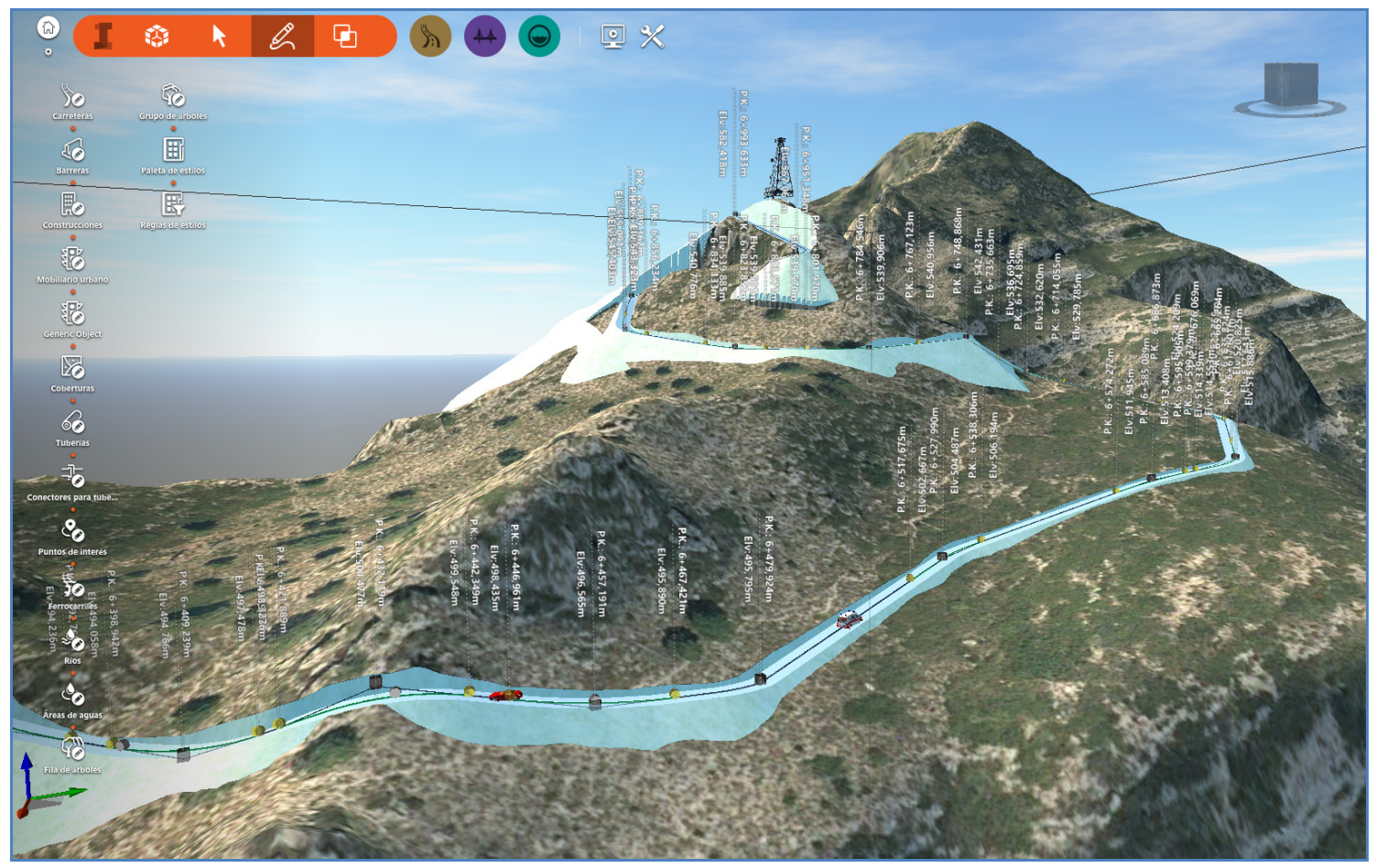

Fig. 2. Vista del desarrollo de las prácticas realizadas mediante el software de realidad virtual Autodesk Infraworks

Es preciso destacar que todo el proyecto se basa tanto en el empleo y procesamiento de datos de libre disposición en repositorios oficiales, como de la aplicación software de libre acceso (QGIS e Infraworks). Se trata, por tanto, de un operativo que pueden utilizar y replicar cuando el alumno acceda a su desarrollo profesional en oficinas técnicas o de proyectos.

\subsection{Implantación en las clases prácticas}

Con objeto de desarrollar la innovación dentro de la asignatura, el alumno trabaja dentro de un grupo el diseño de una vía de acceso a una torre de vigilancia forestal ubicada en un punto de observación dominante, atendiendo a los factores principales que caracterizan el terreno. El profesorado ofrece unas ubicaciones predefinidas para las torres en el sector interior de la provincia de Valencia. Cada ubicación se combina con cuatro alternativas distintas, establecidas por el arranque de la vía de acceso desde dos carreteras diferentes. Estos puntos de inicio están definidos en un intervalo abierto de puntos kilométricos (PKs) entre 2-3 km de longitud, sobre carreteras de la red convencional comarcal, provincial o nacional.

En definitiva, los alumnos quedarán organizados en grupos de 3 o 4 miembros, donde cada grupo tendrá asignado un único punto de destino (la torre). El grupo se reparte entre las dos alternativas ( 1 o 2 alumnos por cada alternativa) según la carretera de inicio. Como no existe un punto concreto de arranque de la obra viaria, cada alumno deberá desarrollar un proyecto personal que no tiene por qué coincidir con el de otro compañero. 
Las actividades concretas desarrolladas en cada clase práctica, y que se deben formalizar y entregar semanalmente adjuntas a las Tareas Poliformat (TP) diseñadas por el profesorado, son:

TP1. INICIO Y OBTENCIÓN DE DATOS: Introducción a QGIS y características básicas. Visualizar localizaciones asignadas con la ayuda del geovisor Terrasit (ICV). Localización y descarga de datos del terreno de libre acceso para el proyecto.

TP2. ORGANIZACIÓN DE DATOS: Definir el recinto de trabajo. Seleccionar y extraer los datos precisos para los proyectos individuales a desarrollar con QGIS.

TP3. TRAZADO ÓPTIMO: Conocer el proceso a desarrollar y los inputs precisos para determinar un trazado óptimo en obras lineales. Ejecutar herramientas SIG específicas de optimización basadas en los factores del terreno disponibles.

TP4. $\quad$ AJUSTE DEL TRAZADO: Adecuar y replantear la traza obtenida a los condicionantes del terreno. Aprovechar y/o evitar sus singularidades. Garantizar la posibilidad real de ejecución de la obra.

TP5. MAPAS DE PROYECTO: Obtener los diferentes mapas básicos del proyecto (situación, localización, trazado) en planta mediante QGIS.

TP6. MODELADO BIM: importar el trazado final replanteado mediante Infraworks. Identificar errores e indefiniciones geométricas procedentes del SIG, corregir el trazado desde el punto de vista geométrico y del aprovechamiento óptimo de los materiales.

Una vez realizadas las anteriores Tareas y completado el calendario de prácticas, el grupo se reúne para escoger y justificar la solución más adecuada. Para ello se tienen en cuenta las características de las alternativas obtenidas por cada miembro del grupo, así como los detalles constructivos principales. Una vez llegado a un acuerdo sobre la alternativa óptima entre las disponibles, se debe elaborar un documento o trabajo final (TF), incluyendo la cartografía aneja específica, de la solución propuesta.

Hay que destacar que cada una de las tareas anteriormente relacionadas recibe una calificación entre 0 y 10 que computa para la nota final de prácticas, de manera que:

$$
\text { Nota Prácticas }=(\mathrm{TP} 1+\mathrm{TP} 2+\mathrm{TP} 3+\mathrm{TP} 4+\mathrm{TP} 5+\mathrm{TP} 6) * 0.1+\mathrm{TF} * 0.4
$$

La calificación de cada Tarea se resuelve mediante asignación de dos revisores (evaluación por pares) realizada automáticamente por Poliformat de entre los alumnos que entregan la Tarea. El profesorado se limita a comprobar que estas calificaciones por pares se ajustan a los criterios establecidos y publicados como referencia a los revisores. El documento final es corregido por el profesorado, y también es puntuado entre 0 y 10 .

En la Fig. 3 siguiente se refleja de forma gráfica el diagrama de flujo de los trabajos a desarrollar por los alumnos que pertenecen a un mismo grupo. 


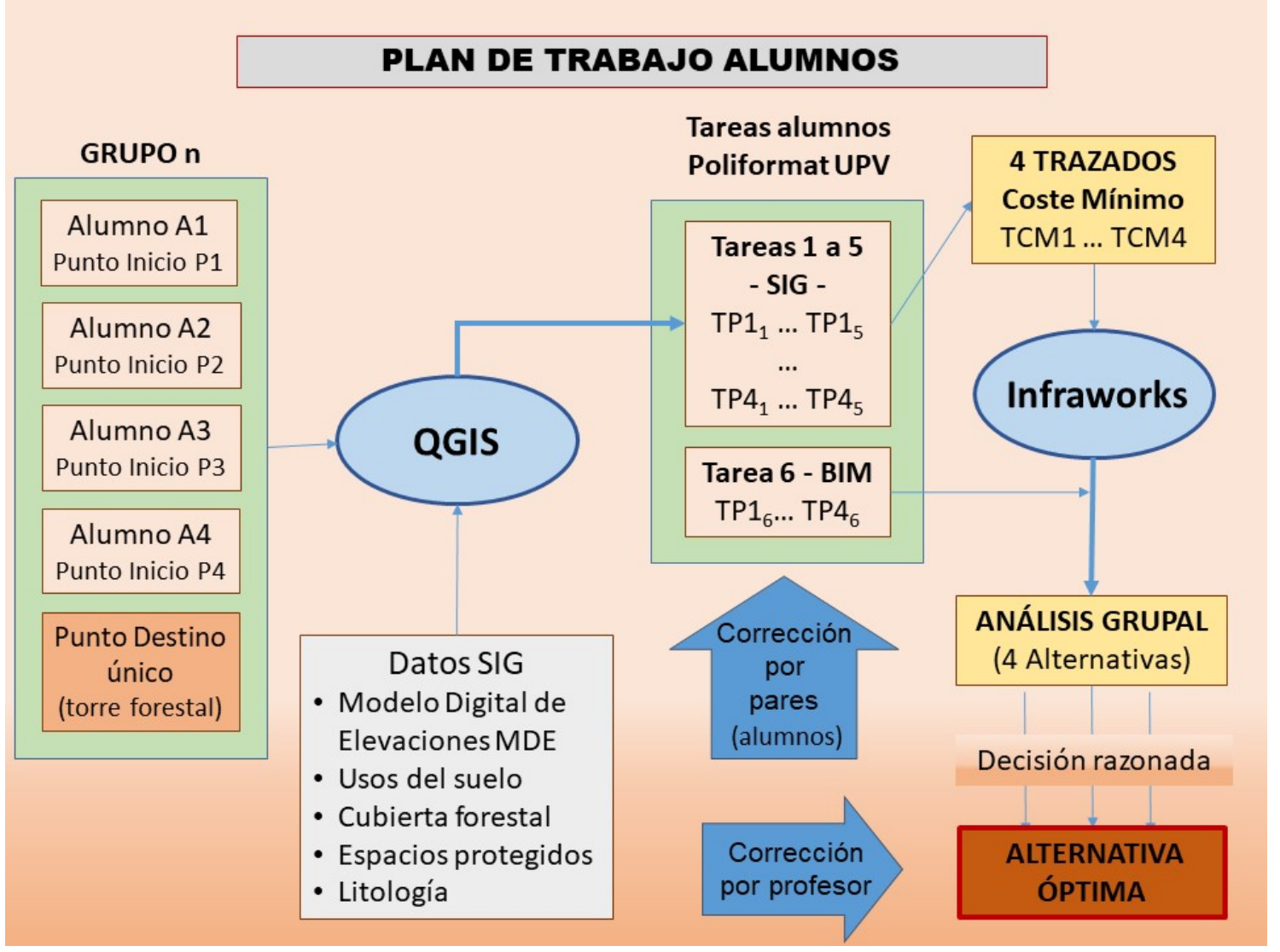

Fig. 3. Esquema del plan de trabajo realizado por los alumnos de la asignatura

\section{Resultados}

En el momento de presentar esta comunicación se dispone de la reciente experiencia del primer cuatrimestre del curso 2019/20. Por tanto, aunque no dejan de ser unos resultados provisionales y parcialmente representativos, tanto los comentarios como la evaluación obtenida por el alumnado señalan la adecuación de la vía iniciada y que, por tanto, precisa consolidar y mejorar.

En efecto, al término de la asignatura se plantearon mediante los sondeos de Poliformat al alumnado unas cuestiones sobre el desarrollo de las prácticas que en este trabajo aparecen detalladas. Sobre una muestra de 25 respuestas (de un total de 26 alumnos matriculados), los resultados se indican en la Tabla 1.

A la vista de los resultados obtenidos a través de esta encuesta, se puede concluir que los alumnos consideran adecuada y de utilidad la innovación, especialmente la aplicación BIM. Plantean reservas en cuanto el empleo de un software libre alternativo como es el QGIS, pero entendemos la gran conveniencia de que conozcan otro software SIG que evita estar sujetos a los altos costes de las licencias comerciales. Por último, los alumnos parecen animar al profesorado a continuar y también mejorar las prácticas sobre el planteamiento iniciado este curso.

Por otra parte la nota media de prácticas de este año ha aumentado en un $5 \%$, siendo en el presente curso de 8,26, frente al 7,83 del curso anterior 2018/19 (sobre 34 alumnos matriculados), donde las prácticas de GIS no se ponían en conexión con la metodología BIM, teniendo esta última una carga docente testimonial. No se dispone de una serie histórica de calificaciones de prácticas de años anteriores que sean comparables, dado que las éstas se modificaron sustancialmente a partir del año 2018-19, y por ello no es 
posible distinguir qué parte del aumento en la nota media registrado este año se debe a la variabilidad del propio grupo, y qué parte es atribuible a la innovación docente. En cualquier caso, la mejora registrada supone un aliciente para continuar durante los próximos cursos con la innovación ahora iniciada.

\begin{tabular}{lr}
\hline 1. ¿Te parecen bien planteadas las prácticas de la asignatura? & $\%$ votos \\
\hline Sí, creo que tienen un desarrollo adecuado y están bien organizadas & 100 \\
Me parecen poco útiles para la asignatura de Ingeniería Civil & 0 \\
Tienen un desarrollo excesivo y me parecen demasiado complejas, se deberían simplificar & 0 \\
\hline 2. ¿Cómo ves el trabajo con un SIG y en concreto con QGIS en la asignatura? & 80 \\
\hline Me parece adecuado trabajar con el software QGIS para completar mi formación SIG & 16 \\
Preferiría trabajar con ArcGIS solamente & 4 \\
Las tareas planteadas son demasiado complejas para resolver con un SIG y sin gran utilidad & \\
\hline
\end{tabular}

3. ¿Cómo ves la aplicación del BIM en la asignatura?

\begin{tabular}{lr}
\hline La encuentro muy apropiada e interesante & 97 \\
No la veo necesaria en esta asignatura & 0 \\
Me parece un software demasiado complejo & 3 \\
\hline
\end{tabular}

\section{4. ¿Cuál es tu opinión sobre el empleo conjunto de SIG y BIM en las prácticas?}

$\begin{array}{lr}\text { Me parece muy adecuado y coherente } & 88 \\ \text { No lo veo necesario para esta asignatura } & 0 \\ \text { Aunque me parece interesante, creo que se deberían realizar modificaciones para mejorar los resultados } & 12\end{array}$

Tabla 1. Resultados encuesta sobre las prácticas de la asignatura (diciembre 2019)

Por último, los docentes decidieron realizar una entrada en LinkedIn (Fig. 4), para observar la respuesta de esta iniciativa docente en el sector profesional y académico. A principios de marzo de 2020, la publicación tuvo un total de 2495 visualizaciones y 31 reacciones, incluyendo empresas e instituciones de reconocido prestigio en los sectores AEC (Architecture, Engineering, Construction) y educativo. Por todo ello, la propuesta docente parece resultar de interés en el ámbito profesional.

Aunque los resultados observados son muy prometedores, la implantación de esta experiencia es muy reciente y precisa un seguimiento estricto para poder mejorar el conjunto, tanto en los documentos de texto como los ámbitos de trabajo propuestos, sin descuidar la simplificación en el manejo del software señalado. El proyecto iniciado es ilusionante para el profesorado y bien considerado por el alumnado. Con estos precedentes, los próximos años confirmarán la consolidación de este proyecto con total seguridad.

Finalmente, el éxito del desarrollo de esta innovación debería marcar solamente el inicio de una tendencia en la escuela, pues el fenómeno BIM no debe quedar constreñido a unas pocas horas dentro de una asignatura generalista. Debe haber una respuesta institucional que garantice unos contenidos más extensos y específicos en esta materia, que potencie una formación BIM pública y de calidad, y claramente demandada por el sector profesional, maximizando así la empleabilidad del alumnado.

\section{Conclusiones}

En esta comunicación se ha detallado la aplicación didáctica integrada de SIG y BIM, llevada a cabo en el curso 2019-2020 como parte de las prácticas de la asignatura Ingeniería Civil, correspondiente al 3er. Curso del Grado de Ingeniería en Geomática y Topografía. 


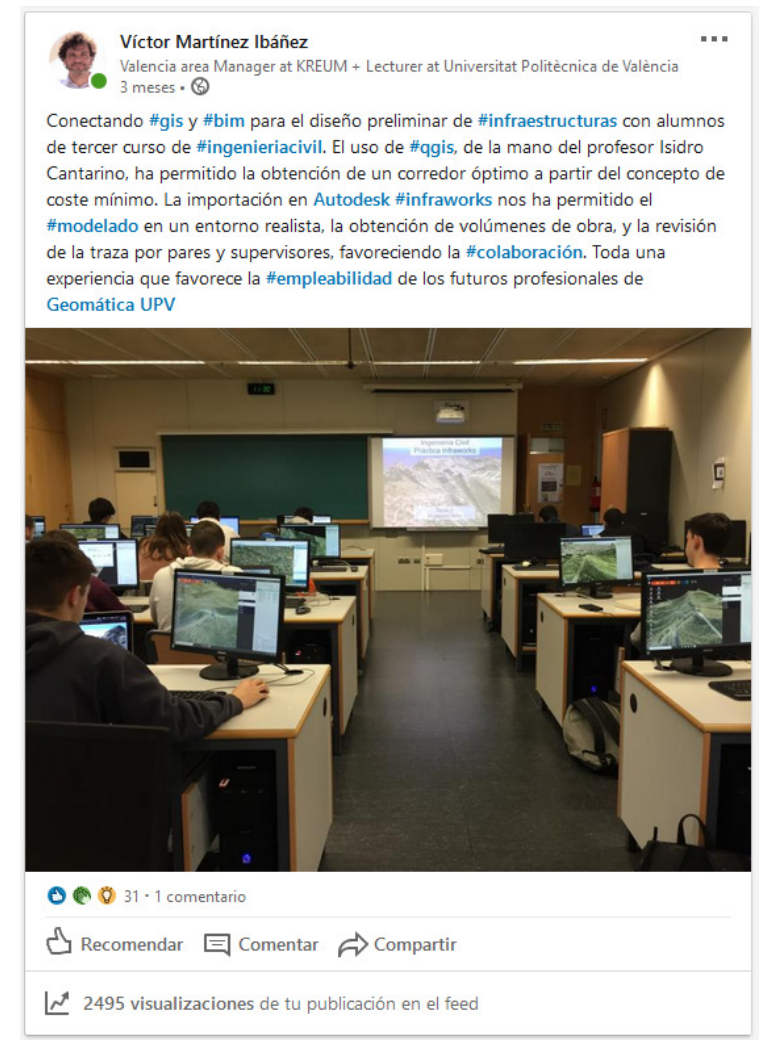

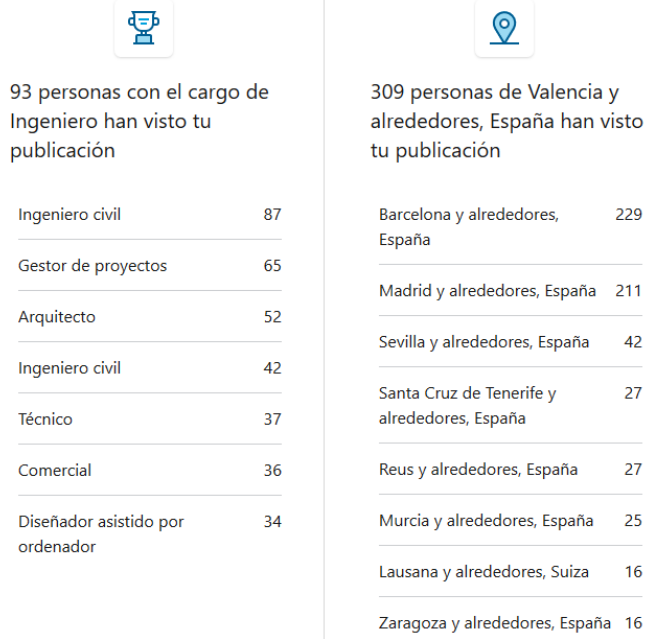

Fig. 4. Publicación en LinkedIn de la iniciativa docente, y datos sobre el impacto provocado (https://www.linkedin.com/posts/vicmarib gis-bim-infraestructuras-activity-6608317120856109056-SvIc)

Esta innovación docente responde a la necesidad de mejorar la total falta de contenidos BIM impartidos en la ETSIGCT, cada vez más presentes en otras Escuelas con formación en el campo de la Ingeniería Civil. Esto es debido a la demanda creciente en el mundo laboral de profesionales formados en este tipo de técnicas.

La experiencia ha permitido mejorar las habilidades de los alumnos en el manejo de herramientas SIG. Los resultados académicos han resultado ser mejores que el curso anterior, por lo que el objetivo planteado parece haber sido alcanzado. Dichos resultados también contribuyen a afirmar que la percepción de las prácticas de la asignatura por parte del alumnado ha mejorado, lo que se atribuye en gran parte al atractivo del software basado en realidad virtual.

Las prácticas planteadas avanzan en dos campos insuficientemente desarrollados en BIM, como es la interoperabilidad entre software de distintas naturalezas, y el empleo de técnicas de realidad virtual en la metodología de diseño de carreteras, lo que permite a los alumnos estar en la punta de lanza de esta nueva tecnología.

La experiencia, que tiene un recorrido todavía muy corto, requerirá de un mayor seguimiento y desarrollo de contenidos por parte de los docentes. Con todo, el impacto en redes sociales apunta al interés por parte del sector AEC y educativo a esta experiencia, y el proyecto iniciado es ilusionante para el profesorado y bien considerado por el alumnado. Por todo lo anterior, incrementar el número de créditos de formación BIM parece una necesidad y una apuesta segura para completar una formación competitiva y actualizada del alumnado de la ETSIGCT, que multiplique la empleabilidad de sus egresados. 


\section{Referencias}

Bradley A, Li H, Lark R, Dunn S (2016) BIM for infrastructure: An overall review and constructor perspective. Autom Constr 71:139-152. https://doi.org/10.1016/j.autcon.2016.08.019

Camacho-Torregrosa FJ, Martínez-Ibáñez V, Aranda-Domingo JÁ, Domingo-Cabo A (2019) Implantación de asignaturas de Building Information Modeling en las titulaciones de la ETSICCP. In: Libro de Actas IN-RED 2019: V Congreso de Innovación Educativa y Docencia en Red. Editorial Universitat Politècnica de València, València

Charef R, Alaka H, Emmitt S (2018) Beyond the third dimension of BIM: A systematic review of literature and assessment of professional views. J Build Eng 19:242-257. https://doi.org/10.1016/j.jobe.2018.04.028

es.BIM (2019) Observatorio de Licitaciones. esBIM 30

Gómez-Muñoz G, Dueñas-Abellán C, Bravo-Bartolomé C, et al (2017) Encuesta de Situación Actual

Gosalves-López J, Murad-Mateu M, Cerdán-Castillo A, et al (2016) Bim En 8 Puntos

Hu M (2019) BIM-Enabled Pedagogy Approach: Using BIM as an Instructional Tool in Technology Courses. J Prof Issues Eng Educ Pract 145:05018017. https://doi.org/10.1061/(ASCE)EI.19435541.0000398

Martinez-Ibañez V, Pellicer E, Alcobendas J, Casado S (2017) Retos en la implantación de BIM en la ingeniería civil y propuestas para acelerar su aplicación. In: Congreso Internacional BIM / 60 Encuentro de Usuarios BIM (EUBIM 2017). Editorial Universitat Politècnica de València, Valencia, Spain, pp 256267

Ministerio de Fomento (2016) Norma 3.1- IC Trazado, de la Instrucción de Carreteras. Gobierno de España. Ministerio de Fomento. Dirección General de Carreteras, Madrid

Ministerio de la Presidencia (2019) Creación de la Comisión Interministerial para la incorporación de la metodología BIM en la contratación pública. Boletín Of del Estado 20648-20659. https://doi.org/10.1017/CBO9781107415324.004

Sidani A, Dinis FM, Sanhudo L, et al (2019) Recent Tools and Techniques of BIM-Based Virtual Reality: A Systematic Review. Arch Comput Methods Eng. https://doi.org/10.1007/s11831-019-09386-0 